\title{
Historiografía de las mujeres en la educación: búsqueda de la mitad perdida
}

\author{
Romelia Hinojosa Luján \\ Profesora investigadora \\ Centro Chihuahuense de Estudios de Posgrado
}

\author{
La historia coloca ante las mujeres un espejo \\ para que ellas se vean a sí mismas \\ y se reconozcan como sujetos sociales \\ Julia Tũ̃ón (2002)
}

\section{Resumen}

$\mathrm{L}$ a presencia de las mujeres ha sido invisibilizada en la historia de México, aunque se trate de la historiografía referida específicamente a un campo eminentemente femenino: el educativo. Constatar qué aspectos, prácticas, temas y referentes contextuales, desde qué elementos metodológicos y teóricos se han sistematizado, es uno de los objetivos de la elaboración de un Estado del Conocimiento de la investigación educativa. El referente geográfico fue el estado de Chihuahua. Fue necesario realizar una investigación hermenéutica para establecer la frontera del conocimiento y poder agendar acciones futuras de investigación.

Palabras clave: historia de la educación, género, investigación histórica, mujeres, investigación educativa.

\section{Introducción}

En Europa y en Estados Unidos la historia con mirada de mujer aparece a principios de los años setenta. Este fenómeno, que causaba cierto escozor, se legitima a partir de dos grandes eventos celebrados en 1973 y 1974 en la ciudad de Berkshire. Conferencias que abordaron sin tapujos la historia de las mujeres de los países europeos.

En México la historia de las mujeres como una disciplina sistemática y profesional cobra vitalidad en la década de los ochenta. En 1988 el Instituto Nacional de Antropología e Historia (INAH) realiza un recuento de la bibliografía publicada sobre la participación de la mujer desde una perspectiva histórica. En él se puntualiza que la mitad de la producción existente de los textos fue escrita a partir de los años setenta, de la cual el $68 \%$ son artí- 
culos; $21 \%$, libros; $5 \%$, tesis; el resto diversos folletos. La temática más abordada es el trabajo femenino, luego la participación social y política de las mujeres. Al revisar a los y las autoras, se encuentra que el 59\% de las obras las habían escrito mujeres; $32 \%$, varones; $7 \%$, diversas instituciones. Dentro de ese porcentaje, 39\% estaba constituido por autoría mixta (Tuñón, 2002). Las cifras anteriores nos brindan un acercamiento a la producción nacional, enfocada al trabajo histórico con mujeres.

El balance que realizamos en este artículo se enfoca en la revisión de investigaciones, libros, capítulos de libros, ponencias, artículos de revista y periodísticos que relatan sucesos históricos cuyo énfasis está puesto en situaciones en relación a la educación de las mujeres en el contexto geográfico chihuahuense. El enfoque metodológico que orientó el análisis e interpretación de la producción realizada en la entidad, fue la hermenéutica.

Si bien las mujeres aparecemos en las fuentes primarias de la historia, nos han invisibilizado en la historiografía. Pareciera como si los participantes y constructores de ella hubiesen sido únicamente hombres. Cuando llegamos a emerger, generalmente lo hacemos gracias y por obra de algún personaje masculino: como madre o esposa, por ejemplo. La historiografía femenina trata de revertir este fenómeno y de rescatar las acciones, vivencias y pensamientos de la otra mitad de los participantes: las mujeres. Pero no solamente es denunciar la falta de visibilidad de las mujeres en los hechos pasados, sino también hacer patente dicho protagonismo: "La historia de la mujer tiene un doble objetivo: restituir a la mujer en la historia y devolver nuestra historia a las mujeres" (Gadol, 1992: 123).

La historiografía feminista, al igual que la perspectiva de género, trabaja dos planos: el académico y el político. "Ante todo, debemos preguntarnos por qué tanta información acerca de las mujeres ha sido olvidada, reprimida u omitida. Y esto vendrá a reanimar la discusión acerca de la relación entre la ciencia (histórica) y la política" (Leydesdorff, 1992: 97).

Este campo de estudio tiene como objeto todos aquellos trabajos historiográficos de reconstrucción que destacan la participación femenina o que las protagonistas son del sexo femenino. "Unir y reconstruir las memorias de las mujeres pueden ser un primer objetivo de la historia femenina, que saque a luz lo que fue suprimido, ocultado y olvidado, llenando así las 'lagunas' de la historia” (Perrot, 1992: $80)$.

Documentar y analizar este campo con la mirada de género en la historia, nos brinda la posibilidad de visibilizar los haceres, pensamientos y creencias de las mujeres chihuahuenses en sus primeras incursiones en la vida pública; analizadas e interpretadas bajo criterios específicos que las historiadoras feministas han ido encontrando. Todas ellas posicionadas en trabajos o funciones del ámbito educativo. Los trabajos encontrados, algunos de forma sencilla y general abonan a todas estas épocas y aportan elementos para reconstruir una historia con la mirada puesta en las mujeres. 


\section{Panorama general del subcampo}

La producción de la investigación histórica de mujeres, en el estado de Chihuahua, está integrada por 21 trabajos de los cuales el $28 \%$ son libros; el $24 \%$, tesis y el $48 \%$, artículos. El $24 \%$ son producciones realizadas por hombres (todas ellas artículos de memoria, periodísticos y de revista) y el 76 \% son de autoría femenina.

Cuatro de los textos analizados son capítulos de libros, compilados con objetos de estudio más amplios, mismos que centraron su mirada en las mujeres. El período de producción de los textos objeto de estudio queda resumido en la gráfica No. 1. la historia de las mujeres, pues carecemos de fuentes escritas sobre muchos aspectos de la vida femenina del pasado" (Leydesdorff, 1992: 95). Es a través de este argumento que nos ofrece Leydesdorff, que podemos entender las razones de una utilización frecuente de archivos orales en los trabajos de historias de mujeres.

A partir del tratamiento que se da a la información recabada y de cómo es concebida la realidad por parte de los y las investigadoras, se percibe, sin tratar de uniformar o someter a algún estereotipo a las investigaciones, una tendencia unánime, tal vez por la naturaleza del objeto de conocimiento, hacia un paradigma cualitativo en la investigación. "La incorporación de la subjetividad del objeto de estudio es un tema que ha sido abordado por sociólogos, antropólogos e historiadores durante décadas, tanto por hombres como por mujeres" (Gordon, 1992: 121).

En torno a la concepción de lo que es la historia o para qué realizar historia, también se aprecian algunas tendencias generalizadas: una de ellas es la historia a partir de la vida cotidiana, otra es la de "dar voz a los sin voz", lema de la
$\mathrm{Al}$ revisar la metodología empleada en estos trabajos, se destacan el método histórico y la historia oral como los más recurrentes. La consulta a la fuente primaria es una pauta notoria en los trabajos; la historia de vida y la entrevista son las técnicas más utilizadas. "Las fuentes orales son muy importantes para historia oral y la microhistoria (historia del terruño o de la "matria") (González y González, 1995). Aunque también se produce un trabajo (Calvo, 2003) en el que se engarzan o analizan los hechos a través de tres tipos de historia: la microhistoria, la historia coyuntural y la historia de las estructuras. 


\section{La educación femenina a través de la historia}

El presente apartado está integrado por las investigaciones históricas que tienen por objeto de estudio la educación de las mujeres y por las mujeres. Los estudios analizados rescatan la otra mitad de la historia.

Chantal Cramaussel (s/f) en "Las mujeres y el papel de la familia en la colonización de la frontera norte de la Nueva España" realiza un estudio amplio en torno a la importancia de la mujer en la expansión colonial. Describe el papel de la mujer, subordinado en la sociedad, por ser la vía de reproducción y expansión de la población en la gran franja fronteriza norte.

En su libro Obedecer, servir y resistir. La Educación de las mujeres en la historia de México, María Adelina Arredondo López (2003) realiza una compilación interesante y diversa de la educación de las mujeres en el siglo XIX. En uno de los capítulos, "Un atisbo a una escuela de niñas al comienzo del siglo XIX", escrito por ella, nos describe el desempeño de la escuela de niñas que funcionó en la Villa de Chihuahua en ese tiempo.

Guillermo Hernández Orozco, en La educación de la mujer en Chihuahua, 1810-1837 (1996), aborda de forma más breve esta temática: demuestra una desigualdad valoral entre la educación que se le ofrece al hombre y la que se le brinda a la mujer. Este autor escribe también una ponencia denominada, “Teoría de género y educación de la mujer en Chihuahua" (2004), en la que reafirma su posición.
Hernández (1996) y Arredondo (2003) coinciden en que la atención que se brindaba a la escuela de hombres era superior a la ofrecida a la institución especialmente dirigida a las niñas.

Por otra parte, Silvia Margarita Martínez Meraz (2006), en el texto Educación y Género. Docencia femenina en Hidalgo del Parral, Chih., retrata la conformación de la escuela de niñas en Hidalgo del Parral. Martínez sugiere a manera de hipótesis que "Cuando se concede graciosamente la necesidad de mejorar la educación femenina formal es para beneficiar a la educación infantil; es decir, a las mujeres para el bien de los "otros" no para el bienestar de ellas mismas” (Martínez, 2006:140). Esta autora ha difundido los hallazgos de su tesis de maestría en varios trabajos (Martínez 2003a; 2003b; 2003c; y 2004).

En esta misma línea: la educación de las mujeres por las mujeres, pero dando un salto temporal de casi cincuenta años, en el texto San Diego de Alcalá: un pueblo que se resiste a morir, Ma. Concepción Franco Rosales (2001) cuenta la microhistoria de un pequeño poblado del municipio de Aldama, Chih. En esta historia trabaja un capítulo sobre la escuela en la que aparecen algunos puntos interesantes: la feminización de la docencia, percibida en el personal que atiende la institución, la modelización que las maestras ofrecían a las niñas y señoritas de la comunidad y por último las acciones de servicio y educación comunitaria con mujeres.

Dentro de la misma temática - la incursión de las mujeres en los espacios educativos 
como alumnas-, encontramos dos textos periodísticos breves pero muy ricos en información y con una gran visión histórica. Jesús Vargas aporta información al tema y a la época a través de sus reflexiones y comentarios. En La mujer y la vida cotidiana en Chihuahua. (Artículo dedicado a Obertina Valdez Obeso como un intento de comprender su paso y su momento en la vida) Vargas (1999) expone una valiente hipótesis en torno a la educación de las mujeres. Sostiene que cuando las mujeres se pudieron educar, cambió el panorama social sobre ellas, su papel y su participación en la vida pública. Vargas lo expresa poéticamente cuando dice: "En el firmamento de Chihuahua se empezó a ver la otra mitad del cielo" (Vargas, 1999: s/p).

En otro artículo llamado Consuelo Uranga. Parte IV, Jesús Vargas Valdez (1995) esboza de forma breve el funcionamiento de la Escuela Industrial para señoritas que era una de las pocas instituciones oficiales que trabajaron en torno a la educación femenina en la ciudad de Chihuahua.

A través de los textos evaluados se aprecian los lentos y paulatinos cambios que se han dado en los contextos históricos que rodeaban a las protagonistas. Si se hace un balance de los periodos históricos analizados, se observa que las investigaciones cubren las épocas que tradicionalmente se han establecido para organizar "la historia". Algunas con mayor profundidad que otras. Sin embargo, no existe una propuesta de ningún tipo para organizar la temporalidad a partir de los cambios producidos en las mujeres y por las mujeres.
Por otro lado estos trabajos realizan una gran contribución al inscribir a la mujer en la historia, pero no se ha escrito la historia de las mujeres. Se les restituye en la historia, pero no se documenta la historia del género, su evolución.

\section{La formación de las maestras}

La formación del profesorado es uno de los temas nodales que han considerado las políticas educativas a lo largo de la historia. Los sistemas, espacios, formas y currículos, han reflejado los cambios que las formaciones económico-sociales le han impuesto a la formación de formadores. En algunos casos, la nueva historia de las mujeres ha documentado los procesos de formación de las docentes y el proceso de feminización de la docencia.

Es interesante entonces analizar las investigaciones históricas que dan cuenta de estos procesos, porque permiten contextualizar las luchas femeninas que tuvieron que librar para abrirse paso en la profesión docente, así como el caso de los estereotipos que dominan el imaginario social en torno a lo que debe ser una educadora, por ejemplo.

Luz Idalia Chacón Rodríguez (2006), en su tesis Reseña de la primera escuela de educadoras 1929-1932, nos da información acerca del primer espacio formal dedicado a la formación de las maestras de educación preescolar: el entonces Instituto Científico y Literario.

Ma. Concepción Franco Rosales (2006) dedica, en su texto Imágenes, voces y recuerdos. Una historia de la Escuela Normal del Estado

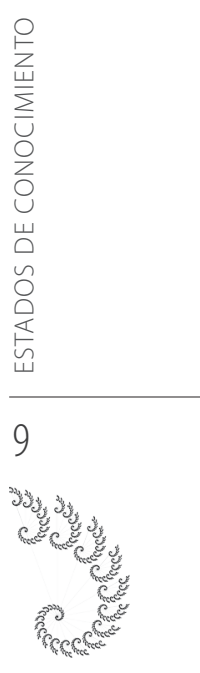


de Chihuahua, un capítulo a la "Formación de educadoras". Documenta también el esfuerzo que la institución realizó para que en 1929 se iniciara con una pequeña generación de la carrera de preescolar. La diferencia principal que se establece entre el trabajo de Franco (2006) y de Chacón (2006) es que la primera abarca un periodo más amplio, mientras que la segunda constriñe su trabajo a tres años.

En otro de los capítulos del texto, Franco (2006) retrata la vida cotidiana del Internado para Señoritas de la ENECH que funciona entre 1956 y 1977. Por el hecho de estar dedicado a las mujeres, su organización denota una tendencia marcada por los estereotipos de género.

En la misma Normal, en la que Franco reseña la existencia del internado y la formación de educadoras, Romelia Hinojosa Luján (2007) realiza un estudio histórico enfocado al plano político denominado "Educación y Política en la formación de maestros. Una historia del Semiescolarizado de la Escuela normal del Estado de Chihuahua". Ella introduce en algunas de las categorías de su tesis, la perspectiva de género como herramienta de análisis de las diferencias culturales que existían entre los y las protagonistas del Semiescolarizado: techo de cristal, masculinización de la imagen directiva, doble jornada, entre otros.

Hinojosa (2007) al igual que Franco (2006) y Martínez (2004) realizan historiografía, pero en determinados momentos escriben focalizando su mirada sobre las mujeres. Incluyen en la historia general a las mujeres y se detienen en el análisis de pautas de género. De esta forma cumplen con uno de los planteamientos que se recomiendan al construir historia con mirada de mujer: evitar caer en el aislamiento o en la separación de la historia de las mujeres. "Es necesario no perderlas en el análisis global pero tampoco, en aras de su rescate, separarlas en forma tajante" (Tuñón, 2002: 391).

A través del balance de este apartado se puede apreciar la historia del proceso de feminización del magisterio: cómo desde un inicio estuvo asociado a la idea de que la mujer podía desempeñarse adecuadamente como docente, "por sus características innatas", o por ser una carrera "cómoda". Algunas mujeres pasaron de forma "natural" a ser maestras, otras, tuvieron que sufrir bastante para poder serlo. El fenómeno de expansión de la docencia como carrera "de mujeres" tiene que ver con la incorporación de mano de obra femenina al sistema de producción asumiendo el papel de "cuidadoras" de niños. Es decir, su incursión en la vida pública se produce supeditada o condicionada a servir a las demás personas. No se hace de forma consciente para posicionarlas profesionalmente, la aceptación es condicionada a coyunturas económicas y sociales que reclaman su trabajo a favor de los y las demás. Sin embargo, el hecho de que paulatinamente muchas de ellas hayan incursionado en la profesión docente sin una formación especializada, devalúa socialmente la imagen y el estatus del profesorado, que se traduce en situaciones de discriminación de sueldos y de condiciones laborales. 


\section{La estela del cometa: la trayectoria de mujeres}

Es común que al hablar de la mujer se realicen generalizaciones. En el ámbito histórico, por ejemplo, se realizan afirmaciones tajantes que desdibujan las características particulares de las mujeres en determinada época. Para contrarrestar lo anterior, donde, bajo la categoría de "mujer", se homogeniza a las mujeres históricamente dadas, que viven en sociedades siempre complejas y enfrentan situaciones contradictorias, se optó por agrupar trabajos en los que se particularizó el ser mujer a través de rostros específicos. Estos trabajos tienen la característica de ser historias de vida de mujeres que, a través de la etapa que les tocó vivir, realizaron trabajos educativos relevantes.

El título de este apartado aluda a la brillantez y trascendencia de las obras que ellas dejaron. Es decir, las historias que en este apartado se trabajan, pueden ser de figuras cotidianas o encumbradas; el hilo que las une, es la aportación de sus obras hacia las mujeres o hacia la sociedad en su conjunto.

Silvia Estrada Erives con la tesis "Hilvanando historias: cinco estudios de caso de maestras originarias del municipio de Guerrero", entreteje o "hilvana" sus historias a través del hilo conductor de ser mujeres de trascendencia educativa que nacieron o laboraron en el municipio de Guerrero, Chih., su trabajo se publicó en un libro titulado Con hilos de colores. Cinco mujeres maestras en el municipio de Guerrero. A través de su texto Estrada (2003) nos transporta a los albores del siglo XX en la puerta a la entrada de la Sierra Tarahumara, mediante la historia de cinco mujeres, maestras destacadas de esta región.

Vargas $(1995,1999)$ dedica dos de sus artículos periodísticos a Consuelo Uranga y a Obertina Valdez Obeso. La primera fue una joven que estudió en la Escuela Industrial para Señoritas. El artículo (Vargas 1995) no ofrece mucha información sobre Consuelo Uranga, no maneja que haya sido maestra y no se puede considerar una historia de vida. Lo incluimos porque alimenta el ansia de rescatar los nombres de las mujeres protagonistas de la historia, lo que las convierte en actores de la misma. Lo mismo sucede con Obertina Valdez de la que sabemos que acorde a los dictados de su época se dio a los y las demás como madre y ama de casa. El relato se realiza a partir de la cotidianidad durante la década de 1920 y 1930.

En la misma línea de este apartado, Silvia Margarita Araiza Mendoza (2008) realiza la investigación "Identidad individual de una maestra rural mexicana. Historia de vida de la Profra. Guadalupe Mendoza Márquez", que también comparte el contexto histórico de algunas de las docentes que retrata Estrada (2004). Araiza (2008) utiliza como categorías de análisis las dimensiones: personal, profesional, pedagógica institucional y social de la identidad. Ella explícitamente dice utilizar la perspectiva de género en el análisis de las dimensiones de la identidad de la maestra Guadalupe Mendoza Márquez. Sin embargo en el análisis del material empírico no se evidencia la perspectiva de género. 
Después de leer los trabajos históricos que reconocen la figura femenina en el contexto de la escuela rural mexicana, nos quedan algunas dudas: ¿Fue igual el apostolado femenino al masculino? ¿Qué funciones, características y condiciones eran diferentes entre las y los docentes? ¿Si se habla de maestra rural de la época, podemos imaginar siempre a una apóstol de la educación?

Desde otras categorías de análisis, Patricia Avitia Estrada en su trabajo "Ser educadora. Testimonios de Josefina La Negra Avitia" (2004), realiza una historia de vida de la maestra Josefina Seáñez de Avitia. Aunque la autora no se posiciona en un método o técnica específica, podemos detectar que a través de la vida cotidiana y de la historia oral, es como ella va entretejiendo la historia de la maestra y su obra educativa.

Es precisamente bajo esta óptica, que se relaciona fuertemente con el estudio "Marina y sus techos de cristal. Las vicisitudes de una maestra", que Beatriz Calvo Pontón realizó en 2003 y que está incluido en el texto Líderes y construcción de poder. Las maestras y el SNTE compilado por Regina Cortina.

Calvo (2003) utiliza como categorías de análisis: la microhistoria, la historia coyuntural y la historia de las estructuras. La microhistoria la usa para delimitar el tiempo corto y las vivencias sucedidas en la inmediatez del mundo empírico y cotidiano de Marina. La coyuntural al relatar procesos sindicales, políticos, partidistas que dieron orientación y sentido específico a la educación y a sus sujetos en el estado de Chihuahua. La histo- ria de las estructuras (aquella que se percibe como inmóvil y de valores prolongadamente fijos) explica con la vida de Marina cómo es "natural" la subordinación de las maestras a los maestros a partir de la historia de la humanidad. Los valores de género que ubican en condiciones de desigualdad a mujeres frente a hombres son estructurales, es decir, prolongadamente fijos.

Esta misma autora también analiza que las relaciones de poder están sujetas a los vaivenes coyunturales; por tanto, cambian, se mueven, adoptan diferentes formas, según sean los momentos (Calvo, 2003).

Queda claro que después de trabajar este apartado, de recorrer historias de protagonistas de la educación chihuahuense, que "las mujeres, como colectivo que vive un momento fundamental de su historia, tenemos necesidad de una memoria que dé cuenta del carácter propio del grupo, de sus procesos y de sus luchas" (Tuñón, 2002: 380).

\section{Balance general}

El hilo conductor que guió este apartado fueron las mujeres en la historia, con la pretensión de dejar de lado su invisibilidad crónica para visibilizarlas y hacerlas patente. Sin embargo, el propósito no es sólo visibilizarnos, sino emprender una búsqueda del sentido de nuestra identidad. Parafraseando a Leydesdorff (1992), la narración entre sí de sus historias ha permitido a las mujeres identificarse unas con otras y han aprendido a comprender que sus problemas no son individuales, por 
lo que han encontrado un terreno común de base para su lucha política.

Este campo ofrece la posibilidad de una construcción fresca e innovadora, por los descubrimientos que aporta, esta es una de sus fortalezas: mucho de lo trabajado en las investigaciones que se analizaron es conocimiento nuevo. Sin embargo, también se tiene que decir que explícitamente tal vez no estaba en los objetivos de las autoras la perspectiva de género, como categoría teórica, que develara la desigual distribución del poder en los hechos que se relatan.

Nos atrevemos a pensar que, en la mayoría de los casos, el trabajo fue realizado a partir de acciones intuitivas, deseos o vivencias personales, más que a partir de un conocimiento profundo de lo que ofrecen las categorías teóricas del género para analizar las relaciones entre hombres y mujeres. De hecho, aunque se tienen incluidas en este campo un número considerable de participantes, no conforman un grupo relacionalmente sólido. Hicieron una historia desde la mirada de lo femenino, descriptiva y sin análisis crítico, más que de una categoría de género, que es una herramienta para denunciar la realidad. La historia desde la categoría de género fundamamenta o cristaliza en la historiografía feminista pues:

...pretende indagar también cómo la feminidad se define desde el estatus de las mujeres, desde la posición y papel que la mujer tiene en una sociedad en relación con la posición y el papel de los hombres. Un enfoque desde esta perspectiva modifica la visión de lo que se entiende tradicionalmente como historia (el ejercicio del poder) y cambia también las premisas de las categorías de análisis histórico, al privilegiar como objeto de análisis la relación entre los géneros (Ramos, 1992: 13).

La historiografía feminista trata de trastocar concepciones previamente aceptadas de los periodos históricos, ha denunciado la idea de que la historia de la mujer es la misma que la del hombre. La historia se podría reescribir y dividirse en periodos de acuerdo con otros momentos cruciales que afectan a la mujer: la sexualidad o estructura familiar, por ejemplo (Gadol, 1992).

Por otro lado, se juzga a las producciones históricas, bajo los moldes de la historiografía masculina. Es decir, el ejemplo a seguir son las producciones hechas por los hombres. Establecemos que podemos utilizar algunos criterios o categorías de análisis extrapolados de la historia masculina, pero no todos. Además de que la historiografía feminista ha propuestos otros criterios o indicadores, que a continuación se destacan:

La historia de las mujeres ha pasado por diversas etapas. Perrot (1992) distingue: Primero el deseo de mostrar que son oprimidas, como lo documentado por Arredondo (2003), Martínez (2006) y Cramaussel (s/f). Segundo, comprender por qué son subyugadas. En el caso de los trabajos analizados no se percibe una incidencia en la búsqueda de explicaciones en este tenor. Tercero, mostrar en todos los niveles su papel positivo, su presencia y participación activa. Son los casos de Estrada 
(2003), Franco (2001y 2006), Araiza (2008), y Avitia (2004). Cuarto, actualmente tiene que ver con el "poder", son los casos de Calvo (2003) e Hinojosa (2007).

Por otro lado, al entrar al debate sobre la existencia o no de una metodología para la historia de las mujeres, podemos encontrar que no existe un método único para tratar la historia de mujeres: la historia oral, el método biográfico, el método histórico, son los más socorridos en las investigaciones que se analizaron en el presente estado de conocimiento.

Sin embargo, se puede apreciar que "La lucha contra las formas existentes de historiografía ha sido desde el principio, después de todo, uno de los objetivos de las historiadoras feministas. Esto ha tenido implicaciones sobre su grado de participación política" (Leydesdorff, 1992: 90). Es decir, aunque las herramientas metodológicas puedan parecer iguales para la historia de las mujeres que para la "general", subyace en la primera la posición política y no sólo académica, de transformación de una realidad injusta en el papel social que se le ha atribuido a la mujer. Por ende, pareciera que de no haber diferenciación en la metodología, la historiografía produce conocimiento semejante en una u otra historia: la participación de las mujeres al hacer historia, dice lo contrario.

Aunque existen los archivos, fuentes y evidencias, siempre destacaron las actividades que los hombres realizan. Pocas veces se descubre la presencia de la mujer: allí estamos. Tal vez porque siempre se acude a fuentes donde nuestra presencia se diluye: los archivos municipales, escolares e institucionales. Es precisamente uno de nuestros planteamientos: imaginar otro tipo de fuentes en las cuales la presencia de las mujeres sea menos sutil (las cartas y los diarios personales, por ejemplo). Un aspecto innovador es la nutrida consulta de fuentes orales que permite recuperar hechos cotidianos de las mujeres que "por carecer de importancia" no hubiesen quedado registrados. "Las fuentes orales son muy importantes para la historia de las mujeres, pues carecemos de fuentes escritas sobre muchos aspectos de la vida femenina en el pasado" (Leydesdorff, 1992: 95).

Es necesario un enfoque histórico que centre el debate en las relaciones sociales entre los géneros, aspecto que está en proceso de incorporación en las investigaciones analizadas, pero que es necesario impulsar fuertemente. Para ello habría que entender las relaciones de género como un proceso, como una construcción social que puede describirse, analizarse en un tiempo y en un espacio determinado, siempre a la vista de éstos y teniendo en cuenta sus modificaciones y sus continuidades.

Específicamente en nuestro campo "El reto está en producir una historiografía que incluya el análisis de género dentro de un cuerpo teórico que explique las desigualdades y diferencias entre mujeres y hombres y no una simple historia descriptiva que lleve por título "Historia de género" y que nos hable de las anécdotas a la manera tradicional" (García, 1998: 228). 


\section{Bibliografía}

Gadol J. (1992) "La relación social entre los sexos; implicaciones metodológicas de la historia de las mujeres en Ramos C. (Comp.) Género e historia, México, Instituto Mora, pp. 123-140.

García, A. (1998). "Historia de las mujeres del siglo XIX: algunos problemas metodológicos”, en E. Bartra, Debates en torno a una metodología feminista, UAM Xochimilco, México.

González y González, Luis (1995) “Pueblo en vilo”, México, El Colegio de Michoacán.

Gordon, L. (1992) “Qué hay de nuevo en la historia de mujeres" en Ramos C. (Comp.) Género e historia, México, Instituto Mora, pp. 110-122.

Leydesdorff, S. (1992). “Política, identificación y la historia de la mujer., en C. Ramos (Comp.), Género e Historia, Instituto Mora/UAM, México, pp. 86-90

Perrot M. (1992) "Haciendo historia: Las mujeres en Francia" en Ramos C. (Comp.) Género e historia, México, Instituto Mora, pp. 66-85.

Ramos, C. (1992). "La nueva historia, el feminismo y la mujer", en C. Ramos (Comp.) Género e Historia, Instituto Mora/ UAM, México, pp. 7-37.

Tuñón, J. (2002). "Las mujeres y su historia. Balance, problemas y perspectiva”, en E. Urrutia (coord.) Estudios sobre las mujeres y las relaciones de género en México: aportes desde diversas disciplinas, COLMEX: Programa Interdisciplinario de Estudios de la Mujer, pp. 375-411

Wallach, J. (1997). “El problema de la invisibilidad”, en Ramos C. (Comp.) Género e Historia, Instituto Mora/UAM, México.

\section{Corpus analizado}

Libros

Franco, M. (2001). "El trabajo de las mujeres, actividad poco valorada”. En Franco M. (ed.), San Diego de Alcalá. Un Pueblo que se resiste a morir. Chihuahua, México: Doble Hélice (pp. 71-83).

Estrada, S. (2004). Con hilos de colores. Cinco mujeres maestras en el municipio de Guerrero. Chihuahua, México: Doble Hélice.

Martínez, S. (2006). Educación y Género. Docencia femenina en Hidalgo del Parral, Chih. (1631-1900). Chihuahua México: Doble Hélice.

Capítulos de libro

Calvo, B. (2003). "Marina y sus techos de cristal. Las vicisitudes de una maestra“. En Cortina R. (comp.), Líderes y construcción de poder. Las maestras y el SNTE, (pp. 67-87). México, D.F.: Editorial Santillana.

Arredondo, A (2003). "Un atisbo a una escuela de niñas al comienzo del siglo XIX”. En Arredondo A. (ed.), Obedecer, servir y resistir. La Educación de las mujeres en la historia de México. México: UPN/Miguel Ángel Porrúa Grupo Editorial (pp. 99-116)

Franco, M. (2001). "El trabajo de las mujeres, actividad poco valorada”. En Franco M. (ed.), San Diego de Alcalá. Un Pueblo que se resiste a morir. Chihuahua, México: Doble Hélice (pp. 71-83).

Franco, M. (2006). “Formación de Educadoras". En Franco M. (ed.), Imágenes, voces y recuerdos. Una historia de la Escuela Normal del Estado de Chihuahua. Chihuahua, Méxi- co: Doble Hélice (pp. 107-118).

Tesis

Araiza, S. (2008). "Identidad individual de una maestra rural mexicana. Historia de vida de la Profra. Guadalupe Mendoza Márquez" (Tesis de Maestría, Centro Chihuahuense de Estudios de Posgrado).

Avitia, P. (2004). "Ser educadora. Testimonios de Josefina "La Negra” Avitia” (Tesis de Maestría, Centro Chihuahuense de Estudios de Posgrado).

Chacón, L. (2006). "Reseña de la primer escuela de educadoras 1929-1932" (Tesis de maestría, Universidad Pedagógica Nacional).

Estrada, S. (2003). "Hilvanando historias: cinco estudios de caso de maestras originarias del municipio de Guerrero" (Tesis de maestría, Centro de Investigación y Docencia).

Hinojosa, R. (2007). "Educación y política en la formación de maestros. Una historia del semiescolarizado de la Escuela Normal del estado de Chihuahua" (Tesis de Doctorado, Universidad de Tijuana).

Informes de investigación

Cramaussel, Ch. (s/f). "Las mujeres y el papel de la familia en la colonización de la frontera norte de la Nueva España“. El Colegio de Michoacán/Centro de Estudios Históricos, sin publicar.

\section{Artículos en periódicos}

Vargas, J. (1995, febrero, 5). “Consuelo Uranga. Parte IV“, en El Heraldo de Chihuahua, pp. 144.

Vargas, J. (1999, abril, 2) "La mujer y la vida cotidiana en Chihuahua", en El Heraldo de Chihuahua, pp. 359.

Vargas, J. (2006, febrero, 12) "La educación porfiriana en el estado de Chihuahua”, en El Heraldo de Chihuahua, pp. 144.

Artículos de revistas

Hernández, G. (1996). “La educación de la mujer en Chihuahua, 1820-1837", en Acoyauh, No. 2, Año 1, noviembre-enero. Órgano de Difusión del Centro de Investigación y Docencia, pp. 18-20.

\section{Publicaciones en memorias}

Hernández, G. (2004). “Teoría de género y educación de la mujer en Chihuahua”, en el 1er. Encuentro Estatal. Experiencias Académicas y de Investigación en Género, pp.16-19.

Martínez, S. (2003a). "Base de datos, tablas y estadísticas de egreso de instituciones formadoras de docentes de Hidalgo del Parral Chih.", en la V Reunión nacional de la red de género en educación, s/p.

Martínez, S. (2003b). “Biografías. Maestras destacadas de Hidalgo del Parral, Chih., en la V Reunión nacional de la red de género en educación, $\mathrm{s} / \mathrm{p}$.

Martínez, S. (2003c). "El proceso de feminización docente en Hidalgo del Parral Chihuahua. Época Colonial I parte., en la V Reunión nacional de la red de género en educación, s/p.

Martínez, S. (2004). "La feminización docente en Parral, Chih., en el 1er Encuentro estatal experiencias académicas y de investigación en género, pp. 8-15.

\section{Notas}

1. Se consideran fuentes primarias a las producidas en la misma época que ocurrió el hecho. Las fuentes secundarias provienen de una época posterior 
